\title{
ESTRUTURA FÍSICO FUNCIONAL DE SERVIÇOS DE ALIMENTAÇÃO COMERCIAL DE UM MUNICÍPIO CENTRAL DO ESTADO DO RIO GRANDE DO SUL ${ }^{1}$
}

\author{
FUNCTIONAL PHYSICAL STRUCTURE OF COMERCIAL FOOD SERVICES
} FROM A COUNTY IN CENTRAL OF THE STATE OF RIO GRANDE DO SUL

\author{
Eluize Pivetta ${ }^{2}$ e Cristiana Basso ${ }^{3}$
}

\section{RESUMO}

O aumento na procura por refeições fora do lar é mais comum em serviços de alimentação comercial que buscam satisfazer o perfil do cliente. O presente estudo teve por objetivos: verificar os setores existentes, comparar dimensionamento das áreas com índices recomendados pela literatura, analisar as vias de acessos e os itens relacionados à ambiência. Tratou-se de uma pesquisa exploratória, com coleta de dados em Serviços de Alimentação Comercial, no período de janeiro a março de 2020, em que aspectos foram obtidos através de observação in loco e plantas baixas. Os resultados referentes a setores e dimensionamentos foram expressos em porcentagem simples. Do montante, foram encontrados nove setores mínimos em todos os estabelecimentos; um estabelecimento apresentou dimensionamento total adequado de acordo com a referência utilizada. Em relação à ambiência, percebeu-se adequação quanto a cores de teto, paredes e piso. No estudo, observou-se que $40 \%$ dos serviços possuem todos os setores, $20 \%$ têm sua dimensão menor que a recomendada e $100 \%$ apresentaram somente duas vias de acesso. Sobre a ambiência, foi encontrada maior inadequação em relação às cores para prevenção de acidentes.

Palavras-chave: Alimentação Coletiva, Serviços de Alimentação, Restaurantes.

\section{ABSTRACT}

The increased demand for outside home meals is more common in Comercial Food Services that targets custommer's satisfying. The present study was a imed to verify the existing sectors, to compare areas' sizingwith indexes recomended by literature, to analyze access routes related to ambience. It was anexploratory research with data collectingon Commercial Food Services between January and March, 2020, Where the aspects were obtained through in loco and floor plan's observation. The results referring to sectors and sizing were expressed in simple percentage. From the amount, were found nine of the minimum sectors in all stablishments, one stablishment presented adequate total dimensioning according to there ference. In relation to the ambience, we have noticed suitability in the color softheceiling, walls and floor. In this work was observed that $40 \%$ of the services have all sectors, $20 \%$ have less dimension than recomended and $100 \%$ have only two access passage ways. About the ambience, was found more inadequation in relation to the colors for accidents' prevention.

Keywords: Collective feeding, Food Services, Restaurants.

\footnotetext{
1 Artigo oriundo de Trabalho Final de Graduação.

2 Nutricionista formada pela Universidade Franciscana. E-mail: eluizepivetta@gmail.com

3 Professora orientadora - Curso de Nutrição - Universidade Franciscana. E-mail: cristiana@ufn.edu.br
} 


\section{INTRODUÇÃO}

Sant'Ana (2012) coloca que a estrutura física de um Serviço de Alimentação (SA), quando bem planejada, tende a satisfazer tanto os clientes que receberão um alimento saudável em um ambiente acolhedor e confortável, quanto os funcionários que terão maior segurança, conforto e motivação para desempenhar suas atribuições. Salienta também, os benefícios à própria empresa com os melhores resultados em produtividade e competitividade no setor de alimentação comercial. Todos esses aspectos podem ser alcançados se houver um trabalho em conjunto de uma equipe multiprofissional, composta por nutricionista, arquiteto, engenheiros e outros profissionais da construção civil responsáveis pela elaboração do projeto.

O ramo de SA está em constante crescimento, com a inclusão de diferentes estabelecimentos, com variedades de refeições, exigindo o engajamento de uma série de profissionais com objetivo em comum de prover alimentos seguros, junto à expansão da empresa (SEBRAE, 2019).

A participação do nutricionista na equipe multiprofissional é prevista de acordo com a lei 8.234/91, que regulamenta a profissão do nutricionista, e com a Resolução CFN 600 de 2018, que coloca como atribuição complementar do nutricionista a participação no planejamento e na supervisão da implantação ou adequação de instalações físicas, equipamentos e utensílios do SA. Nesse planejamento, inclui-se a questão da ambiência, a qual poderá facilitar ou limitar alguns processos na rotina de trabalho (BRASIL, 2018).

Conforme Teixeira (2007), Abreu (2007), Littlefield (2011) e Sant'Ana (2012), no que diz respeito ao porte, aos setores mínimos e dimensionamento coerente dos SA, diversas variáveis devem ser levadas em consideração, dentre elas: o número de refeições, o padrão do cardápio, a modalidade e sistema de distribuição, a política de compras e de abastecimento e os acessos e fluxos da produção. Esses quesitos são importantes para um bom funcionamento do SA e trazem harmonia e agilidade aos serviços prestados.

Consideram-se indispensáveis, independente do porte e tipo do SA, os setores: de recebimento e inspeção, de armazenamento (temperatura ambiente, temperatura controlada, materiais de limpeza), de pré-preparo e preparo, de distribuição, de higienização, de sanitários e vestiários e do administrativo. Setores adequados em relação à separação das atividades e dimensionamento suficiente e coerente proporcionam maior conforto aos funcionários e clientes, diminuem desgastes e melhoram a produtividade e qualidade do produto final (ABERC, 2013).

Para Teixeira (2007) e Neufert (2004), diversas variáveis interferem nos cálculos usados no dimensionamento do tamanho ideal dos setores citados, incluindo o número de refeições no dia ou no maior turno, a quantidade e capacidade dos equipamentos, o contingente de pessoal, o padrão dos cardápios, o número de preparações e complexidade, a quantidade de pessoal e equipamentos necessários, a modalidade e o sistema de distribuição. Além das questões estruturais e 
arquitetônicas do projeto, é necessário perceber que o delineamento do plano de negócio impacta diretamente nas áreas do serviço.

Por exemplo, a política de compras e de abastecimento interfere diretamente na área de armazenamento, já a forma de aquisição dos gêneros alimentícios influencia na área de processamento, pois pode-se adquirir produtos in natura ou pré-processados. O estudo ergonômico também deve ser considerado, porque humaniza a relação gestor/colaborador e reduz licenças de trabalho (MONTEIRO, 2009; PAULA; BIFANO, 2019).

Além da equipe multiprofissional programar os setores que deverão fazer parte do serviço específico e o cálculo exato para dimensionar cada um desses setores, também deverá ser dada atenção especial às entradas e saídas do estabelecimento ou vias de acesso. A recomendação para evitar risco de contaminação cruzada, refere-se, principalmente, à quatro vias de acesso: entrada da matéria-prima, entrada e saída de clientes, entrada e saída de funcionários e saída de resíduos. Entretanto, na impossibilidade de haver essas vias específicas, o problema pode ser minimizado através da via temporal, ou seja, barreira temporal deverá ser respeitada quando horários diferenciados são estipulados para cada acesso (SANT'ANA, 2012).

Outro fator, tão importante quanto à s vias de acesso, refere-se à ambiência, que se caracteriza pelo conjunto de itens que podem interferir na qualidade e quantidade de trabalho produzido, proveniente de condições adequadas em relação à iluminação, cor, ventilação, temperatura, umidade relativa e ruídos (TEIXEIRA, 2007; SANT'ANA, 2012).

Assim, este estudo visa avaliar a estrutura físico-funcional de Serviços de Alimentação Comercial de um município central do Estado do Rio Grande do Sul ao verificar os setores existentes, comparar dimensionamento das áreas com índices recomendados pela literatura, analisar vias de acesso e observar itens relacionados à ambiência.

\section{MATERIAIS E MÉTODOS}

O presente estudo se trata de uma pesquisa exploratória sobre aspectos físico-funcionais de SA situados em um município da região central do estado do Rio Grande do Sul. Foram convidados a participar da pesquisa estabelecimentos que mantivessem vínculo educacional na forma de estágio com a instituição de ensino da pesquisadora, que tivesse nutricionista no local e que disponibilizasse a planta baixa do SA ou condições para a pesquisadora fazer as medidas necessárias. A coleta de dados ocorreu entre janeiro e março de 2020.

Fez-se a análise das plantas baixas e observação in loco dos seguintes itens: áreas/setores existentes, dimensionamento, vias de acesso e ambiência.

Os setores foram analisados por meio da planta baixa e observação in loco. Foram descritos em uma planilha todos os setores que faziam parte de cada SA e depois se comparou com 
a recomendação do que seria o mínimo necessário em relação às áreas, independente de porte e tipo de serviço segundo ABERC (2013).

O dimensionamento dos setores foi verificado por observação das medidas expostas nas plantas baixas quando existentes ou por meio de medição pela própria autora e depois foram organizados em planilha para comparação com índices citados por Teixeira (2007).

Em relação às vias de acesso, foram analisadas a existência das mínimas exigências recomendadas para impedir cruzamentos de fluxo e possíveis contaminações.

Pela observação in loco, tornou-se possível avaliar fatores relacionados à ambiência, detectando adequações ou não quanto à iluminação (se havia luz natural, lâmpadas adequadas e suficientes), cores (índices de reflexão recomendados, tubulações e cores recomendadas pela segurança do trabalho), ventilação, temperatura, umidade relativa (existência de ventiladores, circuladores e exaustores) e ruídos (distância entre paredes paralelas, localização de equipamentos, materiais acústicos e isolantes) (BRASIL, 2004).

Os resultados foram analisados a partir da comparação do existente com a recomendação (Teixeira 2007), utilizando-se de percentual simples efetuado no programa Microsoft Office Excel ${ }^{\circledR}$ versão 2010.

Após a coleta de todos os dados e análise, houve uma devolutiva a cada local participante da pesquisa, com sugestões a respeito de um planejamento físico adequado condizente com suas necessidades particulares.

\section{RESULTADOS E DISCUSSÕES}

Após observação in loco e com auxílio das plantas baixas disponibilizadas pelos cinco SA comerciais analisados e aqui representados por letras, observou-se que todos os estabelecimentos foram definidos como de pequeno porte por não excederem o número de 500 refeições servidas diariamente (SANT'ANA, 2012). Desses cinco, 40\% $(\mathrm{n}=2)$ dispunham de todas as áreas mínimas recomendadas, como demonstrado no Quadro 1.

Quadro 1 - Áreas existentes nos Serviços de Alimentação, Santa Maria/RS, 2021.

\begin{tabular}{|l|c|c|c|c|c|}
\hline \multicolumn{1}{|c|}{ Existência de Setores } & A & B & C & D & E \\
\hline Recebimento e Inspeção & NÃO & SIM & NÃO & NÃO & SIM \\
\hline ArmazenamentoTemperatura Ambiente & SIM & SIM & SIM & SIM & SIM \\
\hline ArmazenamentoTemperatura Controlada & SIM & SIM & SIM & SIM & SIM \\
\hline Armazenamento Materiais de Limpeza & SIM & SIM & SIM & SIM & SIM \\
\hline Pré-preparo & SIM & SIM & SIM & SIM & SIM \\
\hline Preparo & SIM & SIM & SIM & SIM & SIM \\
\hline Distribuição & SIM & SIM & SIM & SIM & SIM \\
\hline Sala Administrativa/ Sala Nutricionista & SIM & SIM & NÃO & NÃO & SIM \\
\hline Higienização & SIM & SIM & SIM & SIM & SIM \\
\hline Sanitários e Vestiários dos Funcionários & SIM & SIM & SIM & SIM & SIM \\
\hline
\end{tabular}

Fonte: Construção do Autor 
Em relação aos setores existentes, observou-se que todos os SA dispunham dos setores de armazenamento (temperatura ambiente e controlada e de material de limpeza), pré-preparo, preparo, distribuição, higienização, sanitários e vestiários.

O setor administrativo não foi encontrado nos Restaurantes $\mathrm{C}$ e D, sendo necessário para uma melhor organização de documentação tanto burocrática como de tabelas e cálculos nutricionais. A necessidade de um espaço físico para esse setor se justifica pela possibilidade de atender fornecedores, funcionários e para realizar pequenas reuniões ou comunicados particulares.

Nos mesmos restaurantes C e D e também no A, não havia o setor de recebimento e inspeção, sendo o trabalho de recebimento desenvolvido no próprio setor de armazenamento. A necessidade de inspecionar a matéria-prima e insumos é de suma importância porque se verifica a qualidade e quantidade dos produtos adquiridos através da verificação de data de validade, condições da embalagem, integridade dos produtos, quantidade solicitada, da temperatura quando necessário, etc.

O estudo bibliográfico de Somavilla e colaboradores (2013), expõe sobre a necessidade de setores bem definidos e organizados, buscando a funcionalidade do processo de produção e assegurando a qualidade do produto. Também, cita a importância do setor administrativo e de recepção e inspeção citados na Legislação Federal pela Resolução da Anvisa RDC 216 de 15 de setembro de 2004 e em nível internacional pelo Codex Alimentarius (Código Alimentar divulgado pela Food and Agriculture Organization - FAO em 1993).

Os SA, em geral, apresentaram boa distribuição segundo as dimensões quando comparadas com as referências, no entanto alguns estabelecimentos apresentaram áreas maiores que o necessário, não sendo um problema, visto que poderá haver expansão da empresa e esses espaços serem oportunamente ocupados por mais equipamentos e utensílios, assim como maior número de funcionários.

Porém, um dos SA apresentou setores com áreas menores; e outro, por fornecer somente refeições transportadas, não possui o salão de refeições, somente a área de distribuição das preparações que serão acondicionadas até seu transporte, conforme o Quadro 2.

Teixeira et al. (2007) utiliza essas quatros áreas (total, total excluindo salão de refeições, armazenamento e produção) para adequação de dimensionamento, que são índices multiplicados pela quantidade de comensais. O autor não estabelece recomendações para as áreas de recebimento, do administrativo, de sanitários e vestiários.

O restaurante A mostrou ser uma empresa que prioriza o conforto com ambientes espaçosos, arejados e sofisticados. A área de produção é o dobro da recomendada, o acesso a subsetores é facilitado e permite-se adquirir equipamentos com maior capacidade caso seja preciso, além de ampliação da equipe de trabalho. 
Quadro 2 - Dimensionamento dos Serviços de Alimentação, Santa Maria/RS, 2021.

\begin{tabular}{|c|c|c|c|c|c|c|c|c|c|c|}
\hline & \multicolumn{10}{|c|}{ ESTABELECIMENTOS } \\
\hline & \multicolumn{2}{|c|}{ A } & \multicolumn{2}{|c|}{ B } & \multicolumn{2}{|c|}{$\mathbf{C}$} & \multicolumn{2}{|c|}{ D } & \multicolumn{2}{|c|}{$\mathbf{E}$} \\
\hline & 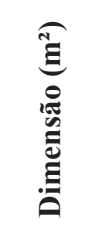 & 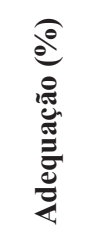 & 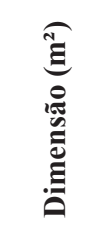 & 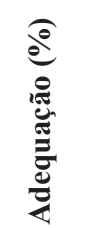 & 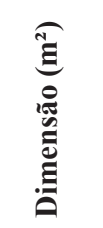 & 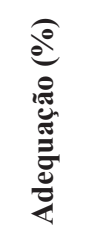 & 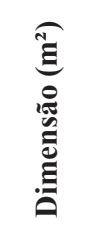 & 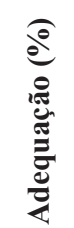 & 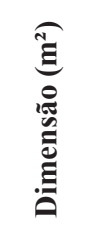 & 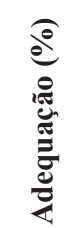 \\
\hline Área Total & 530 & 135,2 & $\mathrm{NA}^{*}$ & $\mathrm{NA}^{*}$ & 226,3 & 57,73 & 300 & 99 & 820 & 160 \\
\hline $\begin{array}{l}\text { Área total excluindo } \\
\text { salão de refeições }\end{array}$ & 222 & 101,9 & 80 & 50 & 115,8 & 53,12 & 171 & 103 & 570 & 203 \\
\hline Área de armazenamento & 26,58 & 66,5 & 20,6 & 98 & 15,25 & 38,12 & 26,2 & 124 & 74,85 & 300 \\
\hline Área de Produção & 60,11 & 200 & 45,22 & 150 & 47,67 & 85,12 & 51,65 & 172 & 125,8 & 167 \\
\hline
\end{tabular}

No estabelecimento B, verificou-se a inexistência de salão de refeições, pois como já mencionado, a distribuição é somente por refeições transportadas e nesse caso a área de distribuição se tornou uma extensão da área de produção, com tamanho suficiente para organização das refeições que serão transportadas.

Quanto ao estabelecimento C, nenhum setor alcançou o tamanho necessário, o que levou a um fluxo confuso, com sobreposição de tarefas, pontos de estrangulamento e movimentação desnecessária de funcionários.

Os estabelecimentos D e E têm áreas maiores que o recomendado. No D, percebeu-se o fácil acesso aos equipamentos e utensílios, já no E, eles estão muito afastados uns dos outros, levando a movimentação supérfluas dos funcionários. Isso somente será justificado se o serviço for ampliado e com isso adquirido novos equipamentos e mais mão de obra.

Em estudo de Macedo et al. (2020), foi calculado o dimensionamento de um SA hospitalar de um município do estado do Maranhão e obtiveram área total de 193,32 m², área de produção 41,46 m² e não há área específica de recebimento. Mezomo (2015) sugere que, para a Unidade de Alimentação e Nutrição (UAN) Hospitalar utilizar $1 \mathrm{~m}^{2}$ multiplicado pela quantidade de leitos, do total, distribuir em porcentagem de $50 \%$ para a produção, $20 \%$ para recebimento e $30 \%$ para demais dependências. Assim, verificou-se a inadequação das dimensões do SA hospitalar do estudo citado.

Sobre as vias de acesso, foram encontradas duas vias em cada estabelecimento, sendo utilizada a barreira temporal para entrada e saída de funcionários e clientes, entrada de matéria prima e descarte de lixo, conforme o Quadro 3.

Sant'Ana (2012) sugere, como forma de evitar cruzamentos, quatro vias de acesso, para entrada e saída de clientes; entrada e saída de funcionários; entrada de matéria prima e insumos; e, saída de lixo. Porém, quando não é possível haver vias específicas, pode-se utilizar barreiras temporais. 
Quadro 3 - Vias de acesso dos Serviços de Alimentação, Santa Maria/RS, 2021.

\begin{tabular}{|l|c|c|c|}
\hline & Via de Acesso 1 & Via de Acesso 2 & Demais acessos \\
\hline Estabelecimento A & Funcionário e Clientela & Matéria Prima e Lixo & - \\
\hline Estabelecimento B & Funcionário, Clientela e Lixo & Matéria Prima & $\begin{array}{c}\text { Saída Lateral - } \\
\text { somente funcionários }\end{array}$ \\
\hline Estabelecimento C & Funcionário e Clientela & Matéria Prima e Lixo & - \\
\hline Estabelecimento D & Clientela & $\begin{array}{c}\text { Funcionário, } \\
\text { Matéria Prima e Lixo }\end{array}$ & Saída de Emergência \\
\hline Estabelecimento E & Clientela & Funcionário, \\
\hline
\end{tabular}

Fonte: Construção do Autor

Corroborando com essa pesquisa, estudo de Macedo et al. (2020), sobre aspectos físicos e funcionais de uma UAN, também verificou que é utilizada a mesma via de acesso para saída de lixo e entrada de gêneros alimentícios, o que propicia o aumento do risco de contaminação cruzada.

No quesito ambiência, todos os SA analisados possuíam iluminação artificial suficiente e todos possuíam proteção contra quedas e explosões. Pohren e colaboradores (2014) fizeram um estudo no qual o objetivo era analisar a estrutura física de uma Unidade de Alimentação e Nutrição e encontraram resultado distinto, em que as lâmpadas não possuíam proteção, trazendo maior risco.

A iluminação deve ser ofertada igualmente entre natural e artificial e deve proporcionar visualização de forma que a tarefa seja efetuada sem prejudicar a higiene e as características sensoriais dos alimentos. Completando, as luminárias devem ser em quantidade adequada e estar protegidas contra explosão e quedas acidentais, de forma que a proteção evite o acúmulo de poeira e gordura oxidada, tornando a limpeza mais fácil (FILHO, 1996; ABERC, 2003; BRASIL, 2004; TEIXEIRA et al., 2007; SANT'ANA, 2012). Quanto às aberturas, somente 20\% $(n=1)$ se apresentou com iluminação natural suficiente e ventilação adequada. Em seu estudo, Fonseca e colaboradores (2010) encontraram somente dois restaurantes adequados na questão ventilação. Essa assegura a renovação do ar e também é importante para manter o conforto térmico, além de facilitar a visualização do alimento, assegurando a higiene e características sensoriais.

Os itens ventilação, temperatura e umidade relativa estão relacionados. A ventilação adequada implica no conforto térmico e proporciona renovação do ar, o que é importante, uma vez que, são exalados continuamente vapores originários da cocção dos alimentos, podendo provocar desconforto térmico. Pode-se assegurar uma ventilação adequada com aberturas de paredes permitindo a movimentação do ar, sendo esta área equivalente a 1/10 da área do piso, havendo a possibilidade de recorrer a meios artificiais como ventiladores, circuladores e exaustores (ABERC, 2013; TEIXEIRA et al., 2007).

Nos Estabelecimentos B e C dos SA, o pé-direito era menor que três metros. Assim, notou-se um maior prejuízo quanto à ventilação, mesmo apresentando o sistema de exaustão com boa funcionalidade. As temperaturas nesses estabelecimentos encontravam-se em $33^{\circ} \mathrm{C}$ e $31^{\circ} \mathrm{C}$ respectivamente dentro da produção. 
A temperatura adequada deve ser entre $22^{\circ} \mathrm{C}$ a $26^{\circ} \mathrm{C}$, com umidade relativa de 50 a $60 \%$, sendo esses valores considerados harmonizáveis para os procedimentos realizados em SA. O sistema de exaustão faz a retirada de gordura, vapor, gases, fumaça, calor e odores, os quais se infiltram em pisos, paredes, tetos e equipamentos, podendo causar incêndio e aumentando o risco de proliferação de bactérias e fungos (TEIXEIRA et al., 2007; SANT'ANA, 2012).

O pé-direito também interfere em uma boa circulação de ar em todos os setores do SA, visto que o uso de equipamentos, iluminação e a cocção dos alimentos geram calor, aconselha-se que o pé-direito deva ter entre 4 e 5 metros, sendo aceitável 3,5 metros (SANTA MARIA, 2018; SANT'ANA, 2012).

Quanto às cores recomendadas, percebeu-se que 100\% (5) das áreas de preparo tinham suas paredes, pisos e tetos na cor branca, em adequado estado de conservação, facilitando o processo de higienização. Resultado semelhante foi encontrado por Costa e colaboradores (2018), em pesquisa feita em uma unidade de alimentação em Bayeux - PB, na qual verificaram os itens teto e piso e encontraram cores claras, materiais de fácil higienização e em adequado estado de conservação.

Contudo, Araújo e colaboradores (2020), em pesquisa que analisou as questões de ambiência em restaurantes comerciais, obtiveram resultados adequados quanto a cores de piso e teto, mas inadequados em relação às paredes.

As cores agem fortemente sobre as pessoas, podendo ocasionar sensações de ânimo, excitação ou relaxamento. Utilizam-se índices de reflexão da luz que são diferentes para teto, $85 \%$ a 100\%; paredes, $50 \%$ a $60 \%$; e pisos, de 30\% a 45\%. De acordo com esses índices, percebe-se melhor adequação com cores claras (TEIXEIRA et al., 2007; SANT'ANA, 2012).

Ainda sobre as cores, todos apresentaram adequação somente na canalização do gás, não havendo identificação em outras canalizações. Em cores para primeiros socorros, apareceu somente o vermelho para prevenção de incêndios, e 20\% $(n=1)$ dos estabelecimentos apresentou cor amarela para cuidado com degraus.

Visto a necessidade de utilização das cores para prevenção de acidentes, recomenda-se o vermelho para identificar perigo presente na área de alarme, hidrantes, extintores e saídas de emergência; amarelo para indicar atenção, usado em parapeitos, corrimões e portas baixas de escada; verde se refere à segurança, empregado em caixas e equipamentos de primeiros socorros, quadro de avisos e cartazes; branco para áreas de bebedouros, armazenagem e zonas de segurança (ABERC, 2013; TEIXEIRA et al., 2007; SANT'ANA, 2012).

Em SA, as cores também se referem a identificação de canalizações, que são o branco para vapor; verde-folha para água fria; laranja para água quente e amarelo ou cinza para gás (ABNT, 1994).

Outro fator que pode atrapalhar o desempenho de atividade no SA se refere a um ambiente de trabalho ruidoso, pois o ruído influencia tanto fisicamente como psicologicamente, podendo levar a sintomas de mal-estar e redução da capacidade de produção (FILHO, 1996; TEIXEIRA et al., 2007). Todos os SA analisados não apresentaram ambiente ruidoso, apesar de nenhum ter isolamento 
acústico. Todos tinham paredes paralelas distantes a menos de 17 metros, o que evita eco, e não tinham grande número de equipamentos industriais, por serem todos os serviços de pequeno porte.

\section{CONCLUSÃO}

No estudo, observou-se que $40 \%$ dos SA possuem todos os setores, $20 \%$ têm sua dimensão menor que a recomendada e 100\% apresentaram somente duas vias de acesso. Sobre a ambiência, foi encontrada maior inadequação em relação às cores para prevenção de acidentes.

Após coleta e análise de todas as informações pertinentes, os locais receberam orientações individualizadas quanto às suas necessidades físicas e funcionais, em que foi reforçada a importância de um planejamento prévio, ou no caso de serviços já existentes, reformas e reparos que tendem a auxiliar em uma melhor estrutura física que contribuirá no alcance das boas práticas.

\section{REFERÊNCIAS}

ABERC - Associação Brasileira de Empresas d e Refeições Coletivas. Manual de práticas de elaboração e serviço de refeições para coletividades. 8 ed. São Paulo: ABERC, 2013. 225 p

ABNT - Associação Brasileira de Normas Técnicas. NBR nº 6.493: Emprego de Cores para Identificação de Tubulações. Rio de Janeiro, 94. Disponível em: https://bit.ly/3v6TcE2. Acesso em 15 out. 2019.

ABREU, E. S.; SPINELLI, M. G.; PINTO, A. M. Gestão de Unidades de Alimentação e Nutrição: Um Modo de Fazer. 2. ed. rev. ampl. São Paulo, SP: Metha, 2007. 318 p

ARAUJO, E. M.; DAMASCENO, B. H.; CARIOCA, A. A. F.; ADRIANO, L. S. Condições de Ambiência em Restaurantes Comerciais. Florianópolis, SC: Revista DEMETRA. v. 15. 2020. Disponível em: https://bit.ly/3fNolpV. Acesso em 14 out. 2019.

COSTA, D. D. C.; LIMA, D. V. T. Análise dos Fatores de Ambiência de uma Unidade de Alimentação e Nutrição da Cidade de Bayeux-PB. Revista Brasileira de Obesidade, Nutrição e Emagrecimento, São Paulo, v. 12, n. 72, p. 522-526, jul/ago. 2018.

FILHO, A. R. A. S. Manual Básico Para Planejamento e Projeto de Restaurantes e Cozinhas Industriais. São Paulo, SP: Livraria Varela, 1996. 232 p 
FONSECA, M. P.; MANFRIDINI, L. A.; SÃO JOSÉ, J. F. B.; TOMAZINI, A. P. B.; MARTINI, H. S. D.;

RIBEIRO, R. C. L.; SANT’ANA, H. M. P. Avaliação Das Condições Físico-Funcionais de Restaurantes

Comerciais para Implantação das Boas Práticas. Revista Alim. Nutr. Araraquara, v. 21, p. 251-257, 2010.

IBGE. INSTITUTO BRASILEIRO DE GEOGRAFIA E ESTATISTICA. Gastos com Alimentação. Disponível em: https://bit.ly/3f1djOv. Acesso em: 23 out. 2019.

LTTLEFIELD, D. Manual do Arquiteto: Planejamento, Dimensionamento e Projeto. 3. ed. Porto Alegre, RS: Bookman, 2011. 767 p

MACEDO, J. L.; OLIVEIRA, A. S. S. S.; PEREIRA, I. C.; FERRAZ, J. R. S.; ASSUNÇÃO, M. J. S. M. Aspectos Físicos e Funcionais de uma Unidade de Alimentação e Nutrição de um Município Maranhense. Palmas, TO: Revista DESAFIOS, v. 7, n. 2, 2020.

MEZZOMO, I. B. Os Serviços de Alimentação: Planejamento e Administração. 6. ed. Barueri, SP: Manole, 2015. 343 p

MONTEIRO, M. A. M. Importância da Ergonomia na Saúde dos Funcionários de Unidades de Alimentação e Nutrição. Revista Baiana de Saúde Pública, v. 33, n. 3, 2009.

NEUFERT, E. Arte de Projetar em Arquitetura: Princípios, Normas, Regulamentos sobre Projeto, Construção, Forma, Necessidade e Relações Especiais, Dimensões de Edifícios, Ambientes, Mobiliário, Objetos. 17. ed. rev. E ampl. São Paulo, SP: Gustavo Gili, 2004. 618 p

PAULA, A. H.; BIFANO, A. C. S. Produções Acadêmicas Brasileiras em Ergonomia e Alimentação Coletiva: Um Estudo Bibliográfico. Revista Brasileira de Engenharia de Produção.

POHEN, N. F. et al. Avaliação da Estrutura Física de uma Unidade de Alimentação e Nutrição. Revista UNIVAP. v. 20. n. 36. 2014.

SANTA MARIA. Lei Complementar nº 119 - Código de Obras e Edificações, de 26 de julho de 2018. Disponível em: https://bit.ly/3vhKtyQ. Acesso em: 14 jul. 2020.

SANT’ANA, H. M. P. Planejamento Físico-Funcional de Unidades de Alimentação e Nutrição. Rio de Janeiro: Rubio, 2012. 288 p 
SEBRAE. Serviço Brasileiro de Apoio às Micro E Pequenas Empresas: Gastos com Alimentação Fora do Lar. Disponível em: https://bit.ly/3f3PeXp. Acesso em 23/10/2019.

TEIXEIRA, S. et al. Administração Aplicada às Unidades de Alimentação e Nutrição. São Paulo: Atheneu, 2007. $219 \mathrm{p}$ 